\title{
Nonessential sum graph of an Artinian ring
}

\author{
Bikash Barman and Kukil Kalpa Rajkhowa \\ Cotton University, Guwahati, India
}

\begin{abstract}
Purpose - The authors study the interdisciplinary relation between graph and algebraic structure ring defining a new graph, namely "non-essential sum graph". The nonessential sum graph, denoted by NES $(R)$, of a commutative ring $R$ with unity is an undirected graph whose vertex set is the collection of all nonessential ideals of $R$ and any two vertices are adjacent if and only if their sum is also a nonessential ideal of $R$.

Design/methodology/approach - The method is theoretical.

Findings - The authors obtain some properties of $\mathrm{NES}(R)$ related with connectedness, diameter, girth, completeness, cut vertex, $r$-partition and regular character. The clique number, independence number and domination number of $\operatorname{NES}(R)$ are also found.
\end{abstract}

Originality/value - The paper is original.

Keywords Nonessential ideal, Nonessential sum graph, Minimal ideal

Paper type Research paper

\section{Introduction}

The growth of interdisciplinary study of graph and algebra took place after the introduction of zero-divisor graph by Istvan Back [1]. Some of the interesting graphs are comaximal graph of commutative ring [2], intersection graph of ideals of rings [3], total graph of commutative ring [4], etc. In [5], Atani et al. introduced a graph associated to proper nonsmall ideals of a commutative ring, namely, small intersection graph. The small intersection graph of a ring $R$, denoted by $G(R)$, is an undirected graph with vertex set is the collection of all nonsmall proper ideals of $R$ and any two distinct vertices are adjacent if and only if their intersection is not small in $R$. Taking this insight of small intersection graph of a ring, we, in this paper, define nonessential sum graph of an Artinian ring.

To continue this sequel, we are going to remember some definitions and notations from ring and graph. Let $R$ be a commutative ring with unity. An ideal $I$ of $R$ is said to be essential in $R$ if $I \cap J \neq 0$, whenever $J$ is a nonzero ideal of $R$. The sum of all minimal ideals of $R$ is known as socle of $R$, denoted by $\operatorname{soc}(R)$. We use $\min (R)$ to denote the collection of all minimal ideals of $R$. The ring $R$ is said to be an Artinian ring if every descending chain of $R$ terminates. In an Artinian ring, every ideal contains a minimal ideal.

Let $G$ be an undirected simple graph with vertex set $V(G)$ and edge set $E(G)$. $G$ is said to be a null graph if $V(G)=\phi$ and that $G$ is said to be empty if $E(G)=\phi$. We denote degree of $v \in V(G)$ by $\operatorname{deg}(v)$. If $\operatorname{deg}(v)=1$, then $v$ is called an end vertex. $G$ is complete if any two vertices are adjacent. $G$ is said to be $r$-regular if degree of each vertex of $G$ is $r$. A walk in $G$ is an alternating sequence of vertices and edges, $v_{0} x_{1} v_{1} \ldots x_{n} v_{n}$ in which each edge $x_{i}$ is $v_{i-1} v_{i}$. A closed walk has the same first and last vertices. A path is a walk in which all vertices are distinct; a circuit is a closed walk which all its vertices are distinct (except the first and last).

\footnotetext{
JEL Classification - 05C25, 13C99, 16D10

(C) Bikash Barman and Kukil Kalpa Rajkhowa. Published in the Arab Journal of Mathematical Sciences. Published by Emerald Publishing Limited. This article is published under the Creative Commons Attribution (CC BY 4.0) licence. Anyone may reproduce, distribute, translate and create derivative works of this article (for both commercial and non-commercial purposes), subject to full attribution to the original publication and authors. The full terms of this licence may be seen at http:// creativecommons.org/licences/by/4.0/legalcode
}

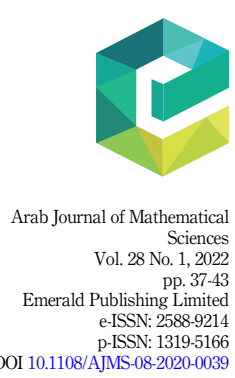

Received 15 August 2020 Revised 22 December 2020 Accepted 22 December 2020 
AJMS 28,1

The length of a circuit is the number of edges in the circuit. The length of the smallest circuit of $G$ is called the girth of $G$, denoted by $\operatorname{girth}(G)$. $G$ is connected if there is a path between every two distinct vertices. $G$ is disconnected if it is is not connected. A vertex of the connected graph $G$ is said to be a cut vertex if removal of it makes $G$ disconnected. If $x$ and $y$ are two distinct vertices of $G$, then $d(x, y)$ is the length of the shortest path from $x$ to $y$ and if there is no such path then $d(x, y)=\infty$. The diameter of $G$ is the maximum distance among distances between all pair of vertices of $G$, denoted by $\operatorname{diam}(G)$. $G$ is said to be a bipartite graph if the vertex set of $G$ can be partitioned into two disjoint subsets $V_{1}$ and $V_{2}$ such that every edge of $G$ joins $V_{1}$ and $V_{2}$. If $\left|V_{1}\right|=m,\left|V_{2}\right|=n$ and if every vertex of $V_{1}$ (or $V_{2}$ ) is adjacent to all vertices of $V_{2}$, then the bipartite graph is said to be complete and is denoted by $K_{m, n}$. If either $m$ or $n$ is equal to 1 , then $K_{m, n}$ is said to be a star. An $r$-partite graph is a graph whose vertex set is partitioned into $r$ subsets with no edge has both ends in any one subset. If each vertex of a partite subset is joined to every vertex that is not in that partite subset, then the $r$-partite graph is said to be complete. A complete subgraph of $G$ is called a clique. The number of vertices in the largest clique of $G$ is called the clique number of $G$, denoted by $\omega(G)$. The neighborhood $N(v)$ of a vertex $v$ in $G$ is the set of vertices which are adjacent to $v$. For each $S \subseteq V(G), N(S)=\cup_{v \in S} N(v)$ and $N[S]=N(S) \cup S$. A set of vertices $S$ in $G$ is a dominating set, if $N[S]=V$. The domination number, $\gamma(G)$, of $G$ is the minimum cardinality of a dominating set of $G$. An independent set of $G$ is a set of vertices of $G$ such that no two vertices are adjacent in that vertex set. The independence number of $G$ is the number of vertices in the largest independence set in $G$, denoted by $\alpha(G)$.

In this paper, we introduce nonessential sum graph of commutative ring with unity. Let $R$ be a commutative ring with unity. The nonessential sum graph of $R$, denoted by $\operatorname{NES}(R)$, is an undirected graph with vertex set as the collection of all nonessential ideals of $R$ and any two vertices $A$ and $B$ are adjacent if and only if $A \cap B$ is also a nonessential ideal of $R$. In this article, we are mainly interested in nonessential sum graph of Artinian ring.

Any undefined terminology can be obtained in [7-8, 15-20].

\section{Connectedness of nonessential sum graph}

In this section, we obtain some results related to connectedness, diameter, girth, completeness, cut vertex, partiteness and regular character. We start with a remark.

Remark 2.1. An ideal $A$ is nonessential ideal in $R$ if and only if $A \subseteq \operatorname{soc}(R)$. If $B$ is a nonessential ideal of $R$ then every ideal which is contained in $B$ is also a nonessential ideal of $R$. If $m$ is a minimal ideal of $R$ and if $A$ and $B$ are two ideals such that $m \subseteq A+B$, then $m \subseteq A$ or $m \subseteq B$.

Lemma 2.2. If $\min (R)=\left\{m_{i}\right\}_{i \in \lambda}$, where $\lambda$ is an index set and $\mu$ is a finite subset of $\lambda$, then $\sum_{\mu} m_{i}$ is a nonessential ideal of $R$.

Proof. If possible suppose $K=\sum_{\mu} m_{i}$ is an essential ideal of $R$. Since each $m_{j} \neq(0)$, so $K \cap m_{j} \neq(0)$ for $j \notin \mu$, which implies that $m_{j} \subseteq K$. But it is a contradiction by Remark 2.1. Hence the lemma.

From this onwards, $R$ is an Artinian ring.

Theorem 2.3. $\operatorname{NES}(R)$ is a null graph if and only if $R$ contains exactly one minimal ideal.

Proof. First consider that $\operatorname{NES}(R)$ is a null graph. On the contrary, assume that $m_{1}$ and $m_{2}$ are two distinct minimal ideals of $R$. So $m_{1} \cap m_{2}=0$ and this provides that both $m_{1}$ and $m_{2}$ are nonessential ideals of $R$, a contradiction. Conversely, suppose that $R$ has exactly one minimal ideal $m$, say. If $m$ is the only nontrivial proper ideal of $R$, then obviously $\operatorname{NES}(R)$ is a null graph. If $A$ is a nontrivial proper ideal of $R$ with $A \neq m$, then it is easy to observe that $A$ is essential in $R$. The proof is complete. 
Theorem 2.4. $\operatorname{NES}(R)$ is an empty graph if and only if $R$ has exactly two minimal ideals, which are the only nonessential ideals of $R$.

Proof. Let NES $(R)$ be an empty graph. Then by Theorem $2.3 \mid \min (R \mid \neq 1$. If $|\min (R)| \geq 3$ and $m_{1}, m_{2}, m_{3} \in \min (R)$, then $m_{1}$ and $m_{2}$ are adjacent by Lemma 2.2. Therefore, $|\min (R)|=2$ and so we take $|\min (R)|=\left\{m_{1}, m_{2}\right\}$ with $m_{1} \neq m_{2}$. Clearly $m_{1}$ and $m_{2}$ are nonessential. If $I$ is any other nonessential ideal which is different from $m_{1}$ and $m_{2}$, then $m_{i} \subset I$ for $i=1,2$. This gives that $I$ and $m_{i}$ are adjacent, a contradiction. Thus $m_{1}$ and $m_{2}$ are the only nonessential ideals of $R$. For the other direction, we consider $R$ has exactly two minimal ideals, which are the only nonessential ideals of $R$. Then $m_{1}+m_{2}=\operatorname{soc}(R)$ is essential. So, $\operatorname{NES}(R)$ is an empty graph. This completes the proof.

Theorem 2.5. The following statements are equivalent:

(1) $\operatorname{NES}(R)$ is disconnected.

(2) $|\min (R)|=2$.

(3) $\operatorname{NES}(R)=G_{1} \cup G_{2}$, where $G_{1}$ and $G_{2}$ are two disjoint complete subgraphs of NES $(R)$.

Proof. $(i) \Rightarrow(i i)$ Suppose that $\operatorname{NES}(R)$ is disconnected. We consider $G_{1}$ and $G_{2}$ are two components of $\operatorname{NES}(R)$ and $I, J$ be two ideals such that $I \in V\left(G_{1}\right)$ and $J \in V\left(G_{2}\right)$. Take the minimal ideals $m_{1}$ and $m_{2}$ with $m_{1} \subseteq I$ and $m_{2} \subseteq J$. If $m_{1}=m_{2}$, then $I-m_{1}-J$ is a path, a contradiction. This asserts that $m_{1} \neq m_{2}$. Again, if $|\min (R)| \geq 3$, then $m_{1}+m_{2}$ is nonessential in $R$. From this we get $I-m_{1}-m_{2}-J$ is a path, a contradiction. Therefore $|\min (R)|=2$.

(ii) $\Rightarrow($ iii $)$ Assume that $|\min (R)|=2$. Then we obtain $\operatorname{soc}(R)=m_{1}+m_{2}$, where $m_{1}$ and $m_{2}$ are the minimal ideals of $R$. Let $G_{i}=\left\{I \subseteq R: m_{i} \subseteq I\right.$ and $I$ is nonessential in $\left.R\right\}$. Let $I$ and $J$ be two nonadjacent vertices in $G_{1}$, then $I+J$ is essential in $R$, which implies $\operatorname{soc}(R) \subseteq I+J$. Hence $m_{2} \subseteq I$ or $m_{2} \subseteq J$, a contradiction because in that case either $I$ is essential or $J$ is essential. So, $G_{1}$ is complete subgraph of $\operatorname{NES}(R)$. In the same way, $G_{2}$ is also a complete subgraph of $\operatorname{NES}(R)$. Suppose $K$ and $L$ are two adjacent vertices where $K \in V\left(G_{1}\right)$ and $L \in V\left(G_{2}\right)$. Since $\operatorname{soc}(R)=m_{1}+m_{2} \subseteq K+L$, so $K+L$ is essential, a contradiction. Thus $\operatorname{NES}(R)=G_{1} \cup G_{2}$, where $G_{1}$ and $G_{2}$ are two disjoint complete subgraphs of $\operatorname{NES}(R)$.

$($ iii $) \Rightarrow(i)$ The proof is obvious.

Theorem 2.6. The diameter of $\operatorname{NES}(R)$ is 1,2 or $\infty$.

Proof. If $\operatorname{NES}(R)$ is disconnected then $\operatorname{diam}(\operatorname{NES}(R))=\infty$. Suppose that $\operatorname{NES}(R)$ is connected. If $I$ and $J$ are two nonadjacent vertices of $\operatorname{NES}(R)$ then $I+J$ is essential in $R$. Consider the minimal ideals $m_{1}$ and $m_{2}$ with $m_{1} \subseteq I$ and $m_{2} \subseteq J$. If $m_{1}+J$ is nonessential, then $I-m_{1}-J$ is a path, which gives $d(I, J)=2$. Similarly, if $m_{2}+I$ is nonessential in $R$, then $d(I, J)=2$. Suppose that $m_{1}+J$ and $m_{2}+I$ are both essential in $R$. Since $\operatorname{NES}(R)$ is connected, so $|\min (R)| \geq 3$. Let $m_{3} \in \min (R)$. Since $I+J$ is essential in $R$, therefore $m_{3} \subseteq I+J$. This implies $m_{3} \subseteq I$ or $m_{3} \subseteq J$. If we take $m_{3} \subseteq I$ then obviously $m_{3}+I$ is nonessential in $R$. We assert that $m_{3}+J$ is nonessential. If possible, $m_{3}+J$ is essential in $R$, then $m_{1} \subseteq \operatorname{soc}(R) \subseteq m_{3}+J$, which gives $m_{1} \subseteq J$. Hence $m_{1}+J=J$ is nonessential, a contradiction. Therefore $I-m_{3}-J$ is a path. Thus $\operatorname{diam}(I, J)=2$.

Theorem 2.7. If $\operatorname{NES}(R)$ contains a cycle, then girth $(\operatorname{NES}(R))=3$.

Proof. First if we consider $|\min (R)|=2$, then by Theorem $2.5 \mathrm{NES}(R)=G_{1} \cup G_{2}$, where $G_{1}$ and $G_{2}$ are two disjoint complete subgraphs of $\operatorname{NES}(R)$. Therefore in this case, girth $(\operatorname{NES}(R))=3$, whenever $\operatorname{NES}(R)$ contains a cycle. Next, when $|\min (R)| \geq 3$, $m_{1}+m_{2}, m_{2}+m_{3}, m_{3}+m_{1}$ are nonessential in $R$ where $m_{i} \in \min (R), i=1,2,3$. Thus $m_{1}-m_{2}-m_{3}-m_{1}$ is a cycle. Hence $\operatorname{girth}(\mathrm{NES})(R)=3$.
Graph of an Artinian ring 
AJMS

28,1
Theorem 2.8. Let $R$ contain finitely many minimal ideals, then the following holds:

(1) There exists no vertex in $\operatorname{NES}(R)$ which is adjacent to every other vertex.

(2) $\operatorname{NES}(R)$ is not a complete graph.

Proof. To prove (i), let $\min (R)=\left\{m_{1}, m_{2}, \ldots, m_{t}\right\}$. Assume that there exists a vertex $I$ in $\operatorname{NES}(R)$ such that $I$ is adjacent to every other vertex. Let $m_{i} \subseteq I$ for some $i$. Let $K=\sum_{j \neq i} m_{j}$, which is nonessential in $R$. Thus $K$ is a vertex in NES $(R)$. Now, $K+I \supseteq \sum_{j \neq i}+m_{i}=\operatorname{soc}(R)$. Hence $K+I$ is essential, a contradiction to the fact that $I$ is adjacent to every other vertex. Hence the result.

(ii) Clearly $\operatorname{NES}(R)$ is not complete by (i).

Theorem 2.9. If $\operatorname{NES}(R)$ is connected, then $\operatorname{NES}(R)$ has no cut vertex.

Proof. On the contrary assume that $I$ is a cut vertex of $\operatorname{NES}(R)$. Then $\operatorname{NES}(R) \backslash\{I\}$ is disconnected. Thus, there are vertices $J$ and $K$ with $I$ lies in every path joining $K$ to $J$. By Theorem 2.6, $d(K, J)=2$ and therefore $J-I-K$ is a path. We claim that $I$ is a minimal ideal of $R$. If not, there exists an ideal $L$ of $R$ such that $L \subseteq I$. As $I$ is nonessential in $R$, therefore $L$ is also nonessential in $R$. Since $J+L \subseteq J+I$ and $J+I$ is nonessential in $R$, so $J+L$ is nonessential in $R$. In the same direction, $K+L$ is also nonessential in $R$. So, $J-L-K$ is a path in $\operatorname{NES}(R) \backslash\{I\}$, which is a contradiction. Thus, $I$ is a minimal ideal of $R$. Now, we assert that there exist a minimal ideal $m_{i} \neq I$ of $R$ such that $m_{i} \nsubseteq J$. If not then $m_{i} \subseteq J$ for each $I\left(\neq m_{i}\right) \in \min (R)$ and so $\sum_{m_{i} \neq I} m_{i} \subseteq J$. This gives that $\operatorname{soc}(R)=I+\sum_{m_{i} \neq I} m_{i} \subseteq I+J$, a contradiction to the fact that $I+J$ is nonessential. Similarly, there exists $m_{j}(\neq I)$ such that $m_{j} \nsubseteq K$. Now we see that for each $m_{t} \in \min (R)$ either $m_{t} \subseteq J$ or $m_{t} \subseteq K$. Since $J+K$ is essential, $m_{t} \subset \operatorname{soc}(R) \subseteq J+K$, which implies $m_{t} \subseteq J$ or $m_{t} \subseteq K$. Let $I \neq m_{i}, m_{j} \in \min (R)$ such that $m_{i} \nsubseteq J$ and $m_{j} \nsubseteq K$. Therefore, $m_{i} \subseteq K$ and $m_{j} \subseteq J$. So, $K-m_{i}-m_{j}-J$ is a path in $\operatorname{NES}(R) \backslash\{I\}$, a contradiction. Therefore, $\operatorname{NES}(R)$ has no cut vertex.

Theorem 2.10. $\operatorname{NES}(R)$ is not a complete $r$-partite graph.

Proof. If possible assume that $\operatorname{NES}(R)$ is a complete $r$-partite graph with $r$ parts $V_{1}, V_{2}, \ldots, V_{r}$. Since two minimal ideals are always adjacent, by Remark 2.1, so each $V_{i}$ contains at most one minimal ideal. Thus we get $|\min (R)| \leq r$. Our claim is $|\min (R)|=r$. Suppose $\min (R)=\left\{m_{1}, m_{2}, \ldots, m_{t}\right\}$ and $t<r$. Without loss of generality we can take $m_{i} \in V_{i}$ for $1 \leq i \leq t$. So, $V_{t+1}$ contains no minimal ideal. Since $\min (R)$ is finite, so $\sum_{j \neq i} m_{j}$ is nonessential in $R$. Now, $\sum_{j \neq i} m_{j}+m_{i}=\operatorname{soc}(R)$, so $\sum_{j \neq i} m_{j}$ and $m_{i}$ are not adjacent. Thus $\sum_{j \neq i} m_{j} \in V_{i}$ as $m_{i} \in V_{i}$. Let $I \in V_{t+1}$ and $m_{k} \subseteq I$ for some $m_{k} \in \min (R)$. So, $I$ is adjacent to $m_{k}$. Since $\operatorname{NES}(R)$ is assumed to be complete r-partite and $m_{k} \in V_{k}$, so $I$ is adjacent to every element of $V_{k}$, which implies $I$ is adjacent to $\sum_{i \neq k} m_{i}$, a contradiction. Therefore, $|\min (R)|=r$. Now, consider $J=\sum_{i=3}^{r} m_{i}$. Clearly $J$ is nonessential in $R$ by Remark 2.1. As $J$ is adjacent to $m_{1}$ and $m_{2}$, so $J \notin V_{1}, V_{2}$. Moreover, $J+m_{i}=J$ for $3 \leq i \leq r$. So, $J$ is adjacent to all minimal ideals of $R$. We get that $J \notin V_{i}$ for each $i$, a contradiction. Hence the theorem.

Theorem 2.11. The following statements holds:

(1) $\operatorname{NES}(R)$ contains an end vertex if and only if $\operatorname{NES}(R)=G_{1} \cup G_{2}$, where $G_{1}$ and $G_{2}$ are two disjoint complete subgraphs of $\operatorname{NES}(R)$ and $\left|V\left(G_{i}\right)\right|=2$ for some $i=1,2$.

(2) $\operatorname{NES}(R)$ is not a star graph.

Proof. (i) Let $I$ be an end vertex of $\operatorname{NES}(R)$. So, $\operatorname{deg}(I)=1$. Suppose $|\min (R)| \geq 3$. For each $m_{i} \in \min (R), m_{i}$ is adjacent to every other minimal ideal of $R$, so $\operatorname{deg}\left(m_{i}\right) \geq 2$. Hence $I$ is not a 
minimal ideal. We can assume $m_{1} \subseteq I$. Hence $I$ and $m_{1}$ are adjacent. Since $\operatorname{deg}(I)=1$, so the only vertex adjacent to $I$ is $m_{1}$ and $m_{j} \nsubseteq I, j \neq 1$. Again $I$ and $m_{2}$ are not adjacent, so $I+m_{2}$ is essential. So we get, $m_{j} \subseteq \operatorname{soc}(R) \subseteq I+m_{2}$ for $j \neq 1$, which implies $m_{j} \subseteq I$ for $j \neq 1$, a contradiction. So, $|\min (R)|=2$. By Theorem 2.5, $\mathrm{NES}(R)=G_{1} \cup G_{2}$, where $G_{1}$ and $G_{2}$ are two disjoint complete subgraphs of $\operatorname{NES}(R)$. Let $I \in V\left(G_{i}\right)$. Since $G_{i}$ is a complete subgraph and $\operatorname{deg}(I)=1$, so $\left|V\left(G_{i}\right)\right|=2$. The converse part is clear.

(ii) Suppose that $\operatorname{NES}(R)$ is a star graph. So, $\operatorname{NES}(R)$ contains an end vertex. By the previous part $|\min (R)|=2$ and then by Theorem 2.5, the graph is disconnected. Hence, $\operatorname{NES}(R)$ is not a star graph.

Theorem 2.12. The following statements holds:

(1) If $I$ and $J$ are two vertices of $\operatorname{NES}(R)$ such that $I \subseteq J$, then $\operatorname{deg}(I) \geq \operatorname{deg}(J)$.

(2) If $\operatorname{NES}(R)$ is an $r$-regular graph then $|V(\operatorname{NES}(R))|=2 r+2$.

Proof. (i) Suppose $I$ and $J$ are two vertices of $\operatorname{NES}(R)$ such that $I \subseteq J$. Let $K$ be a vertex adjacent to $J$. So, $J+K$ is nonessential in $R$. As $I+K \subseteq J+K$, so $I+K$ is nonessential in $R$. Thus, each vertex adjacent to $J$ is also adjacent to $I$. Hence $\operatorname{deg}(I) \geq \operatorname{deg}(J)$.

(ii) Let $\operatorname{NES}(R)$ be an $r$-regular graph. So, for each $m_{i} \in \min (R), \operatorname{deg}\left(m_{i}\right)=r$. Since $m_{i}$ is adjacent to each minimal ideal, by Remark 2.1, so $\min (R)$ is finite. Suppose, $|\min (R)| \geq 3$, so $\operatorname{deg}\left(m_{1}+m_{2}\right) \leq \operatorname{deg}\left(m_{1}\right)$ by (i). Also, $\operatorname{deg}\left(m_{1}+m_{2}\right) \neq \operatorname{deg}\left(m_{1}\right)$, since $\sum_{j \neq 2} m_{j}$ is adjacent to $m_{1}$ but not to $m_{1}+m_{2}$. Thus, $\operatorname{deg}\left(m_{1}+m_{2}\right)<\operatorname{deg}\left(m_{1}\right)$, a contradiction. So, $|\min (R)| \leq 2$. If $|\min (R)|=1$ then $\operatorname{NES}(R)$ is null. Therefore, $|\min (R)|=2$. By Theorem 2.5, $\operatorname{NES}(R)=$ $G_{1} \cup G_{2}$, where $G_{1}$ and $G_{2}$ are two disjoint complete subgraphs of $\operatorname{NES}(R)$. Let $\min (R)=\left\{m_{1}, m_{2}\right\}$ and $m_{1} \in G_{1}$. Since $\operatorname{deg}\left(m_{1}\right)=r$, so $\left|G_{1}\right|=r+1$. In the same direction, $\left|G_{2}\right|=r+1$. Hence, $|V(\operatorname{NES}(R))|=2 r+2$.

\section{Clique number, independence number, domination number of nonessential sum graph}

In this section, we will find clique number, independence number, domination number of $\operatorname{NES}(R)$.

Theorem 3.1. The following holds:

(1) $\omega(\operatorname{NES}(R)) \geq|\min (R)|$.

(2) If $\omega(\operatorname{NES}(R))<\infty$, then number of minimal ideals of $R$ is finite.

(3) $\omega(\operatorname{NES}(R))=1$ if and only if $\min (R)=\left\{m_{1}, m_{2}\right\}$ and these two are the only nonessential ideals in $R$.

(4) If the number of minimal ideals of $R$ is finite, then $\omega(\operatorname{NES}(R)) \geq 2^{|\min (R)|-1}-1$.

Proof. (i) Since any two minimal ideals of $R$ are adjacent, by Lemma 2.2, the subgraph with vertex set $\left\{m_{i}\right\}_{m_{i} \in \min (R)}$ of $\operatorname{NES}(R)$ is complete. So, $\omega(\operatorname{NES}(R)) \geq|\min (R)|$.

(ii) If $\omega(\operatorname{NES}(R))<\infty$, then by (i) the number of minimal ideals of $R$ is finite.

(iii) It is clear from Theorem 2.4 .

(iv) Let $\min (R)=\left\{m_{1}, m_{2}, \ldots, m_{t}\right\}$ and for each $1 \leq i \leq t$, take $A_{i}=\left\{m_{1}, m_{2}, \ldots\right.$, $\left.m_{i-1}, m_{i+1}, \ldots, m_{t}\right\}$. Let $P\left(A_{i}\right)$ be the power set of $A_{i}$. For each $X(\neq\{\}) \in P\left(A_{i}\right)$, consider $R_{X}=\sum_{T \in X} T$. Clearly $T_{X}$ is nonessential. Also, subgraph with vertex set $\left\{R_{X}\right\}_{X \in P\left(A_{i}\right)}$ is a complete subgraph which is clear by Lemma 2.2. Now, $\left|P\left(A_{i}\right) \backslash\{\}\right|=2^{|\min (R)|-1}-1$. Therefore $\left|\left\{R_{X}\right\}_{X \in P\left(A_{i}\right)}\right|=2^{|\min (R)|-1}-1$. Hence, $\omega(\operatorname{NES}(R)) \geq 2^{|\min (R)|-1}-1$.
Graph of an Artinian ring 
AJMS

28,1

Theorem 3.2. The following holds:

(1) $\gamma(\mathrm{NES}(R)) \leq 2$.

(2) $\min (R)$ is finite if and only if $\gamma(\operatorname{NES}(R))=2$ and $\min (R)$ is infinite if and only if $\gamma(\mathrm{NES}(R))=1$.

Proof. (i) Since $\operatorname{NES}(R)$ is not a null graph, $|\min (R)| \geq 2$. Consider $T=\left\{m_{1}, m_{2}\right\}$, where $m_{1}, m_{2} \in \min (R)$. Take a vertex $I$ in $\operatorname{NES}(R)$. If $m_{1} \subseteq I$ or $m_{2} \subseteq I$, then $m_{1}+I$ or $m_{2}+I$ is non-essential in $R$. Then $I$ is adjacent to $m_{1}$ or $m_{2}$. Suppose that $m \nsubseteq I$ and $m \nsubseteq J$. If $I$ is not adjacent to $m_{1}$, then $m_{1}+I$ is essential in $R$. So, $m_{2} \subseteq \operatorname{soc}(R) \subseteq m_{1}+I$, which implies $m_{2} \subseteq I$, a contradiction. Therefore $I$ is adjacent to $m_{1}$. In the same way, $I$ is adjacent to $m_{2}$. Thus $\gamma(\operatorname{NES}(R)) \leq 2$.

(ii) If $\min (R)$ is finite, then by Theorem 2.8 , there exists no vertex which is adjacent to every other vertex. So, $\gamma(\operatorname{NES}(R)) \neq 1$. Therefore, $\gamma(\operatorname{NES}(R))=2$ by part (i). In the opposite direction, let $\gamma(\operatorname{NES}(R))=1$. So, the graph has a vertex which is adjacent to every other vertex. So the graph does not contain finite minimal ideals. Hence the result.

Theorem 3.3. Let $R$ contain finite number of minimal ideals. Then $\alpha(\operatorname{NES}(R))=|\min (R)|$.

Proof. Let $\min (R)$ be finite and $\min (R)=\left\{m_{1}, m_{2}, \ldots, m_{t}\right\}$. Since $\left\{\sum_{j=1, j \neq 1}^{t} m_{j}\right\}_{i=1}^{t}$ is an independent set in $\operatorname{NES}(R)$, therefore $t \leq \alpha(\operatorname{NES}(R))$. Assume that $\alpha(\operatorname{NES}(R))$ is equal to $p$ and $S=\left\{I_{1}, I_{2}, \ldots, I_{p}\right\}$ is the maximal independent set. For each $I \in S, I$ is nonessential in $R$. So, there exists a minimal ideal $m$ such that $m \nsubseteq I$. If $p>t$, then there exists $1 \leq i, j \leq p$ and $m \in \min (R)$ such that $m \nsubseteq I_{i}$ and $m \nsubseteq I_{j}$. Thus $m \nsubseteq I_{i}+I_{j}$. Otherwise, $m \subseteq I_{i}+I_{j}$ which leads to a contradiction. As $S$ is independent, so $I_{i}+I_{j}$ is essential, which implies $m \subseteq \operatorname{soc}(R) \subseteq I_{i}+I_{j}$, a contradiction. Therefore, $\alpha(\operatorname{NES}(R))=|\min (R)|$. If we take $\alpha(\operatorname{NES}(R))=\infty$, then by similar argument we get a contradiction. Hence, $\alpha(\operatorname{NES}(R))=|\min (R)|$.

\section{References}

[1] Beck I. Coloring of commutative rings. J Algebra. 1988; 116: 208-226.

[2] Sharma PK, Bhatwadekar SM. A note on graphical representation of rings. J Algebra. 1995; 176(1): 124-127.

[3] Chakrabarty I, Ghosh S, Mukherjee TK, Sen MK. Intersection graphs of ideals of rings. Discrete Math. 2009; 309(17): 5381-5392.

[4] Anderson DF, Badawi A. The total graph of a commutative ring. J Algebra. 2008; 320: 2706-2719.

[5] Atani SE, Hesari SDP, Khoramdel M. A graph associated to proper non-small ideals of a commutative ring. Comment Math Univ Carolin. 2017; 58(1): 112.

\section{Further reading}

[6] Atiyah MF, Macdonald IG. Introduction to commutative algebra, London: Addison-Wesley; 1969.

[7] Balkrishnan R, Ranganathan K. A text book of graph theory. New York, NY: Springer-verlag, Reprint; 2008.

[8] Goodearl KR. Ring theory, Marcel Dekker; 1976.

[9] Harary F. Graph theory, Reading, Mass: Addison-Wesley Publishing Company; 1969.

[10] Haynes TW, Hedetniemi ST, Slater PJ (Eds). Fundamentals of domination in graphs, New York, NY: Marcel Dekker; 1998.

[11] Huckaba JA. Commutative rings with zero-divisors, New York, Basel: Marcel-Dekker; 1988. 
[12] Kaplansky I. Commutative rings, revised ed., Chicago: University of Chicago Press; 1974.

[13] Kasch F. Modules and rings, Academic Press (London); 1982.

[14] Lambeck J. Lectures on rings and modules, Waltham, Toronto, London: Blaisdell Publishing Company; 1966.

\section{Corresponding author}

Kukil Kalpa Rajkhowa can be contacted at: kukilrajkhowa@yahoo.com 